The general principles of neutron activation analysis and the availability of this service suggest that the method may be of value to public health workers.

\title{
Neutron Activation Analysis
}

\author{
By MORGAN S. SEAL, M.S., WILLIAM A. MILLS, M.S., and \\ JAMES G. TERRILL, Jr., C.E., M.B.
}

$\mathrm{P}$ UBLIC HEALTH laboratories may in the near future put to use a nucleonic method of identifying and measuring trace quantities of elements. In essence, the process consists of exposing a sample of the material to be analyzed to neutrons in a reactor. The elements in the sample thereby become radioactive and their radiations provide the key to their character and abundance. In most instances, this service may be obtained through contract with reactor operators.

The detection and quantitative estimation of trace quantities of metallic contaminants in such materials as water, milk, food, insecticides, and construction materials have always presented problems to the public health analyst. The magnitude of these problems depends on the specificity and sensitivity of standard analytical procedures available for each individual contaminant and, to a certain extent, on the medium in which the contaminant is found.

Recent developments in electrometric, spectrophotometric, and spectrographic techniques

Mr. Seal is a chemist and Mr. Mills is a physicist with the Radiological Health Program, Division of Sanitary Engineering Services, Public Health Service. Mr. Terrill is chief of the program.

G. W. Leddicotte, chief of the activation analysis group at the Oak Ridge National Laboratory, and his staff assisted the authors in making a study of the neutron activation method of analysis. They contributed their time, facilities, materials, and knowledge. have greatly increased the number of methods available to the chemical analyst and, in addition, because of the greater specificity of the new techniques, have shortened the time and reduced the labor required for most of the classic gravimetric and volumetric analytical procedures. Continued application of instrumental methods of analysis by public health workers should be encouraged through the development of applied research programs designed to solve specific public health problems. At the same time, public health personnel may want to consider the possibilities of using neutron activation analysis.

The theory of chemical analysis by the identification of characteristic radiations emitted by a particular nuclear species was advanced soon after the discovery of artificial radioactivity, and the technique was first applied to the solution of analytical problems in 1936 (1). Before the development of the nuclear reactor, however, these analyses necessitated the use of particle accelerators or other sources of highly energetic, charged nuclear particles. The scope of such analyses was necessarily limited by the low particle beam intensities.

In theory, any nuclear reaction producing a radioactive isotope that may be identified by its characteristic radiation can be used for activation analysis. However, the most useful reaction involves the capture of a neutron by a stable nucleus to produce a radioisotope of the original atom. The radioisotope formed will have a characteristic nuclear emission of a par- 
ticular energy or energies. In the most frequently used type of reaction, the nuclear emission is a gamma $(\gamma)$ ray, and reactions of this type are commonly designated as $(n, \gamma)$ reactions. A generalized reaction of the $(n, \gamma)$ type is:

$$
{ }_{a} X^{b}+{ }_{o} n^{1} \rightarrow{ }_{a} X^{b+1}+\gamma
$$

In this reaction, the elemental isotope $(X)$ is bombarded by a neutron $(n)$, resulting in a radioactive isotope of the element $(X)$ and an instantaneous gamma ray $(\gamma)$. Decay of the radioactive isotope may result in a characteristic gamma ray. It is the characteristic gamma ray of the radioactive isotope that is of importance in this method, not the gamma from the $(n, \gamma)$ reaction.

Modern radiation-detection instruments are capable of detecting gamma radiation of a particular energy and of differentiating it from gamma radiations of other energies. The amount of gamma activity is dependent on the quantity of radioactive nuclei present. In addition, each radioisotope decays at a constant rate. By observing the amount of gamma activity at two or more suitable time intervals, the decay rate may be ascertained. These quantities, the energy and the decay rate, are normally sufficient to determine exactly what nuclear species are present; the activity then determines the number of radioactive atoms present.

Neutron activation analysis has been carried out on an ever-increasing scale at the Oak Ridge National Laboratory since 1943. Since it is not the purpose of this paper to review the theory or the history of neutron activation analysis, the reader is referred for this information to the excellent unclassified report entitled "Neutron Activation Analysis" by Leddicotte and Reynolds (2).

\section{General Procedure}

The sample containing the contaminant may be either a solid or a solution. In either case, a known amount by weight or by volume is placed in a suitable container for insertion in a nuclear reactor. A pure weighed sample (analytical reagent grade will usually suffice) of the element to be determined, known as the standard, is also placed in the container, as close to the unknown as possible. The container is usually made either of quartz or aluminum, substances which do not shield the samples from the neutron field and which do not in themselves present an excessive radiation hazard after neutron bombardment. For the sake of accuracy, two or more samples of both the unknown and the standard are prepared.

The standard may be either the element itself, as is the case with copper determinations, or a stable compound, such as $\mathrm{Na}_{2} \mathrm{CO}_{3}$ for sodium determinations. The use of a thermally stable chemical form of the standard is important, since the samples are often subjected to considerable heat. More important, however, it is desirable for safety reasons that the elements in the standard other than the element being determined not form intensely radioactive isotopes.

The size of the samples and standards depends on the rate of formation of the radioisotope in a particular neutron field. The rate of formation may be calculated from the rate of decay, which, as stated above, is a constant for a particular radioisotope. In addition, the known intensity of the neutron field and the probability that a particular atom will capture a neutron enter into the calculation. The probability that a certain reaction between a nucleus and an incident particle or photon will occur is known as the nuclear cross section of the atom. These factors may be summarized in the following equation:

where

$$
\mathrm{W}=\frac{A M}{6.02 \times 10^{23} f \sigma S}
$$

$W=$ weight of the element

$A=$ activity of disintegrations per second

$M=$ chemical atomic weight

$6.02 \times 10^{23}=$ Avagadro's number

$$
\begin{aligned}
& f=\begin{array}{l}
\text { neutron intensity (flux) per } \mathrm{cm}^{2} \text { per } \\
\text { second }
\end{array} \\
& \sigma=\begin{array}{c}
\text { probability of neutron capture (cross } \\
\text { section) in } \mathrm{cm}^{2}
\end{array} \\
& S=\text { saturation factor }
\end{aligned}
$$

The factor $S$ warrants further explanation.

$$
S=1-e^{\frac{-0.693 t}{T_{3 / 3}}}
$$


or the ratio of the amount of activity produced in time $t$ to that produced in infinite time. The half-life $\left(T_{1 / 2}\right)$ is determined experimentally for each isotope. It may be obtained or calculated from any compilation of nuclear data, such as the Seaborg tables (3).

The actual irradiation is carried out in a nuclear reactor at a point where the desired neutron intensity (flux) is available. The neutron flux need not be constant, since both the unknown and the standard are subjected to the same flux during the same length of time. The sample is allowed to remain in the reactor until the desired amount of radioactivity is obtained, as calculated from the above equation. After this length of time, the container is removed from the reactor, and the samples are prepared for radiochemical separation, due precaution being observed to protect the worker from radiation exposure.

No specific instructions can be given for the preparation of the sample for radiochemical analysis, except to state that the sample must be put into solution. Procedures vary with such substances as plant material, animal tissue, and other matter. Care must be taken to prevent the loss of easily volatilized radioelements if they are being determined. Dissolution of the standard should present no problem since the standard selected will be readily soluble.

The validity of a radiochemical analysis depends on the fact that radioactive atoms behave exactly the same way chemically as nonradioactive atoms of the same element. Although a large amount of radiation may appear in a particular sample, the actual weight of radioactive material present is extremely minute, often being in the order of $10^{-10} \mathrm{gm}$., or even less. For this reason, it is usually necessary to add to the dissolved unknown measured quantities of pure, standardized, nonradioactive solutions of the element being determined. Such materials are known as carriers, since the relatively few radioactive atoms are carried with the milligram quantities of nonradioactive material throughout the radiochemical analysis. Recovery of the total amount of carrier in the final weighing form need not be quantitative, but it should be in the order of 60 percent or better.

The procedure for chemical analysis depends on the element being determined. Kahn has prepared abstracts of unclassified radiochemical methods that cover the literature through 1953 (4). In addition, members of the Analytical Chemistry Division of the Oak Ridge National Laboratory are preparing an unclassified "master manual" of radiochemical techniques.

\section{An Example: Copper Determination}

To obtain more information on activation analysis, the authors, with the assistance of G. W. Leddicotte and the activation analysis group at the Oak Ridge National Laboratory, carried out three separate analyses: the determination of copper in a nickel oxide sample, the determination of copper in an animal tissue sample, and the determination of sodium in a cast iron sample. We selected these analyses because they present examples of a contaminant in both organic and inorganic materials and because they illustrate the differences between elements. Details of the experiment are given only for the determination of copper in nickel oxide.

\section{Irradiation and Chemical Analysis}

Triplicate 100-mg. samples of nickel oxide and a single $20-\mathrm{mg}$. sample of pure copper metal were irradiated for 16 hours in the Oak Ridge National Laboratory's graphite reactor. Neutron flux at the point of irradiation equaled about $6.50 \times 10^{11}$ neutrons per $\mathrm{cm}^{2}$ per second.

To separate the copper from the other elements, we followed the procedure set down by the laboratory's Analytical Chemistry Division, as given below :

1. To the acid solution of the irradiated sample, add standardized copper carrier and the following holdback carriers: iron, cobalt, zinc, manganese, cadmium, strontium, and sodium.

2. Adjust the solution to $1 \mathrm{~N} \mathrm{HCl}$, saturate with sodium sulfide, centrifuge, and discard supernate. Wash precipitate with two $10-\mathrm{ml}$. portions of hot water. Centrifuge and discard washes.

3. Dissolve the precipitate in $1 \mathrm{ml}$. of concentrated $\mathrm{HNO}_{3}$ containing $\mathrm{BrO}_{3}$, dilute to 10 ml. with $\mathrm{H}_{2} \mathrm{O}$, and add Fe holdback carrier. Make basic with $6 \mathrm{~N} \mathrm{NH}_{4} \mathrm{OH}$, and centrifuge out $\mathrm{Fe}(\mathrm{OH})_{3}$. Add additional Fe holdback carrier, and centrifuge down on top of first $\mathrm{Fe}(\mathrm{OH})_{3}$ precipitate. Discard precipitate. 
Table 1. Results of copper determination

\begin{tabular}{|c|c|c|c|c|c|}
\hline Sample & $\begin{array}{l}\text { Net weight } \\
\text { (mg.) }\end{array}$ & Dilution & $\begin{array}{c}\text { Separated } \\
\text { CuCNS } \\
\text { weight } \\
\text { (mg.) }\end{array}$ & $\begin{array}{l}\text { Percent } \\
\text { yield }{ }^{1}\end{array}$ & $\begin{array}{l}\mathrm{CCM}^{2} \text { (counts } \\
\text { per minute) }\end{array}$ \\
\hline 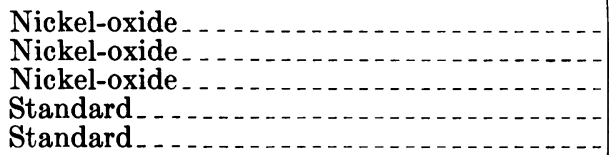 & $\begin{array}{l}\text { 52. } 4 \\
\text { 43. } 7 \\
\text { 46. } 2 \\
\text { 41. } 0 \\
\text { 41. } 0\end{array}$ & $\begin{array}{c}1: 1 \\
1: 1 \\
1: 1 \\
1: 10^{4} \\
1: 10^{4}\end{array}$ & $\begin{array}{l}\text { 19. } 1 \\
22.2 \\
27.1 \\
27.2 \\
21.9\end{array}$ & $\begin{array}{l}\text { 40. } 3 \\
\text { 46. } 8 \\
\text { 57. } 2 \\
\text { 57. } 4 \\
\text { 46. } 2\end{array}$ & $\begin{array}{l}41,032 \\
37,233 \\
43,443 \\
99,763 \\
79,881\end{array}$ \\
\hline
\end{tabular}

1 Theoretical yield of CuCNS was $47.4 \mathrm{mg}$.

2 Count corrected for background and chemical yield.

4. Acidify with $\mathrm{HCl}$ to $0.5 \mathrm{~N}$ in $\mathrm{HCl}$. Add sodium bisulfite solution to reduce $\mathrm{Cu}^{++}$to $\mathrm{Cu}^{+}$, and add solid KCNS until CuCNS precipitates; then add slight excess of KCNS. Centrifuge and wash precipitate with two $10-\mathrm{ml}$. portions of water.

$\check{5}$. Filter with suction through a tared filter paper. Wash with three 5-ml. portions of water and three 5-ml. portions of alcohol. Dry 15 minutes at $110^{\circ} \mathrm{C}$. Weigh as CuCNS (1.92 mg. per mg. $\mathrm{Cu}$ ). Mount and count.

\section{Calculating the Quantity}

After chemical separation, each sample was counted on a scintillation counter, using a thallium-activated sodium iodide crystal, an RCA 5819 photomultiplier, and an aluminium absorber for stopping soft beta rays. (Other equally suitable counting methods are available.) From the radioactivity in the standard

Figure 1. Calibration spectrum of $\mathrm{Na}^{22}$

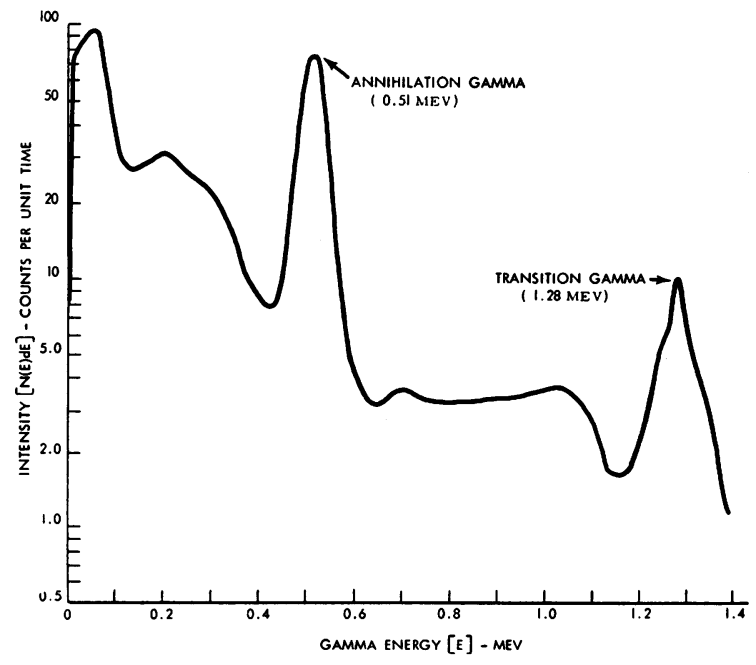

samples and that in the nickel oxide samples, the trace quantity of copper was determined. The data for the samples are given in table 1.

Calculations for the determination of copper were based on the following relationships :

Percent chemical yield $=$ $\frac{\text { weight of } \mathrm{CuCNS} \text { recovered }}{\text { theoretical yield of } \mathrm{CuCNS}} \times 100$.

Count corrected for background $(\mathrm{CCB})=$ CCM-background per minute.

Count corrected for chemical yield $(\mathrm{CCY})=$ CCB

percent chemical yield

CCY for unit weight of $\mathrm{Cu}$ in standard $=$ CCY $\times$ dilution weight of standard sample

Calculation of $\mathrm{Cu}$ in unknown sample: CCY for $\mathrm{Cu}$ in $X$ sample

(weight of $X$ sample) (CCY for unit weight of $\mathrm{Cu}$ in standard)

$=$ number of grams of $\mathrm{Cu}$ in $X$ sample.

Results of the calculations gave an average value of $4.21 \times 10^{-5} \mathrm{gm}$. $\mathrm{Cu}$ per gram of nickel oxide.

The purity of radioactive copper in the samples was checked in two ways.

First, we checked the radioactive half-life by re-counting each sample after an appropriate time interval. The decay agreed within 3 percent of the 12.8-hour half-life of the $\mathrm{Cu}^{64}$ isotope.

Second, we examined one of the standard samples and one of the unknown samples on a single-channel gamma ray scintillation spectrometer to measure the energies of the gamma rays emitted from $\mathrm{Cu}^{64}$. The gamma rays measured were those resulting from annihila- 
tion of the positive electron (positron) and the emission of the rare-occurring ( 0.5 percent) 1.34-Mev. gamma from the $\mathrm{Cu}^{64}$ isotope. When a positron emitter decays, the positron and an electron will collide, annihilate their masses, and produce, usually, two gammas of $0.51 \mathrm{Mev}$. each. (The energy associated with the rest mass of an electron or positron is 0.51 Mev.) The scintillation spectrometer was calibrated with a known source of $\mathrm{Na}^{22}$, which is also a positron emitter, in addition to having a transition gamma. Figures 1, 2, and 3 show the results from the spectrometer. Since a gamma peak for all samples occurred at 0.51 Mev., the purity of the copper in the test samples was satisfactory.

\section{Limitations}

Iike any other method of analysis, radioactivation analysis has its limitations. These limitations may be divided into three classes: physical, chemical, and nuclear (5).

\section{Physical}

1. A sample must be of such character as to withstand temperatures of possibly $70^{\circ} \mathrm{C}$. to $90^{\circ} \mathrm{C}$.

Figure 2. $\mathrm{Cu}^{64}$ spectrum: standard sample.

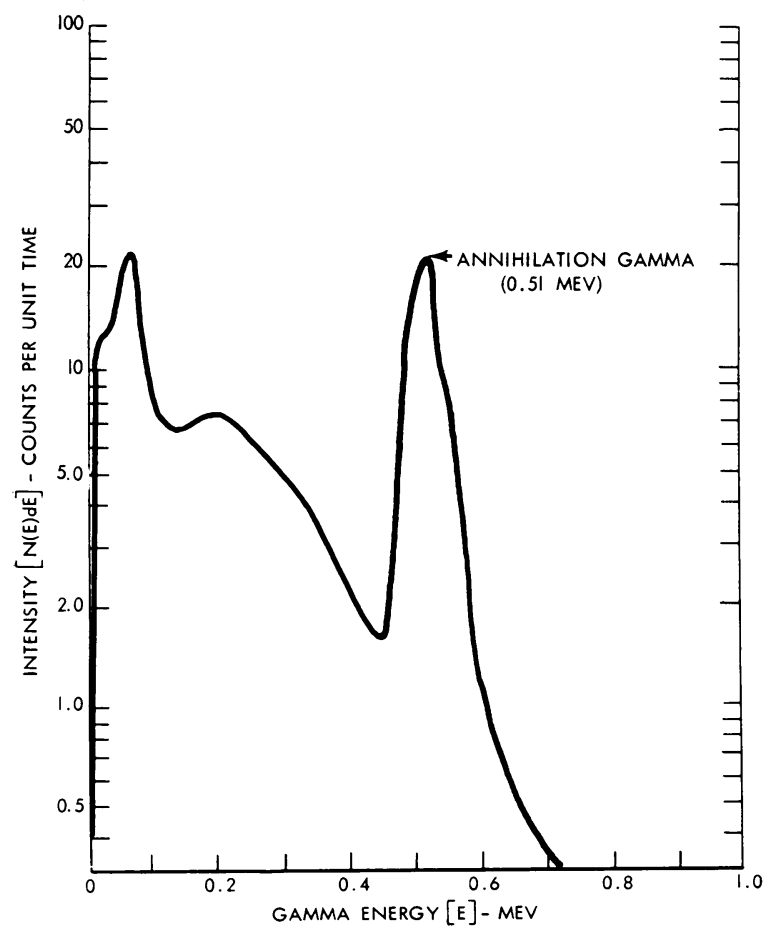

Figure 3. $\mathrm{Cu}^{6+}$ spectrum: nickel oxide sample.

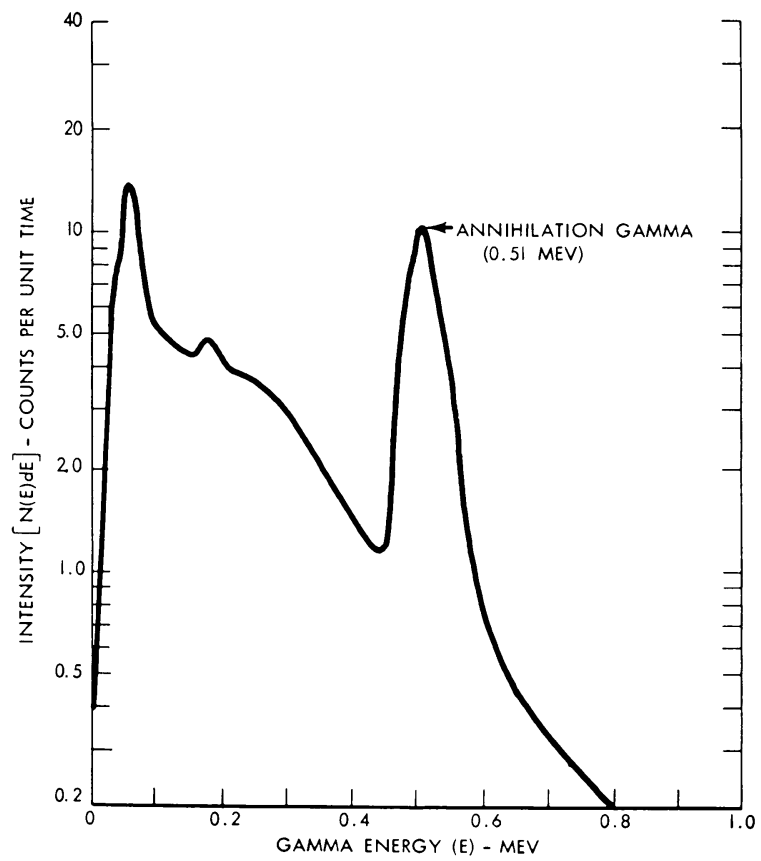

2. Containers must be constructed of materials that, when irradiated, have very short half-lives.

3. The size of the samples is limited by the irradiation space in a reactor.

\section{Chemical}

1. Samples may undergo structural changes as a result of high radiation intensities, and these changes may result in losses of radioactive material by volatilization.

2. Some limitations arise in separating the desired activity from other radioactive elements in the samples, the magnitude of these limitations depending on the number of steps in the radiochemical procedure used.

3 . It is not always easy to treat the samples in such manner that the irradiated element and the carrier element will behave similarly chemically.

\section{Nuclear}

1. 'The samples must not have such a high neutron absorption as to affect the neutron flux throughout the samples.

2. A major limitation is that the isotope formed may be the result of neutron reactions other than an $(n, \gamma)$ reaction.

3. Of some importance is the formation of radioisctopes of the same element differing from 
Table 2. Sensitivity comparison (micrograms per millilifer)

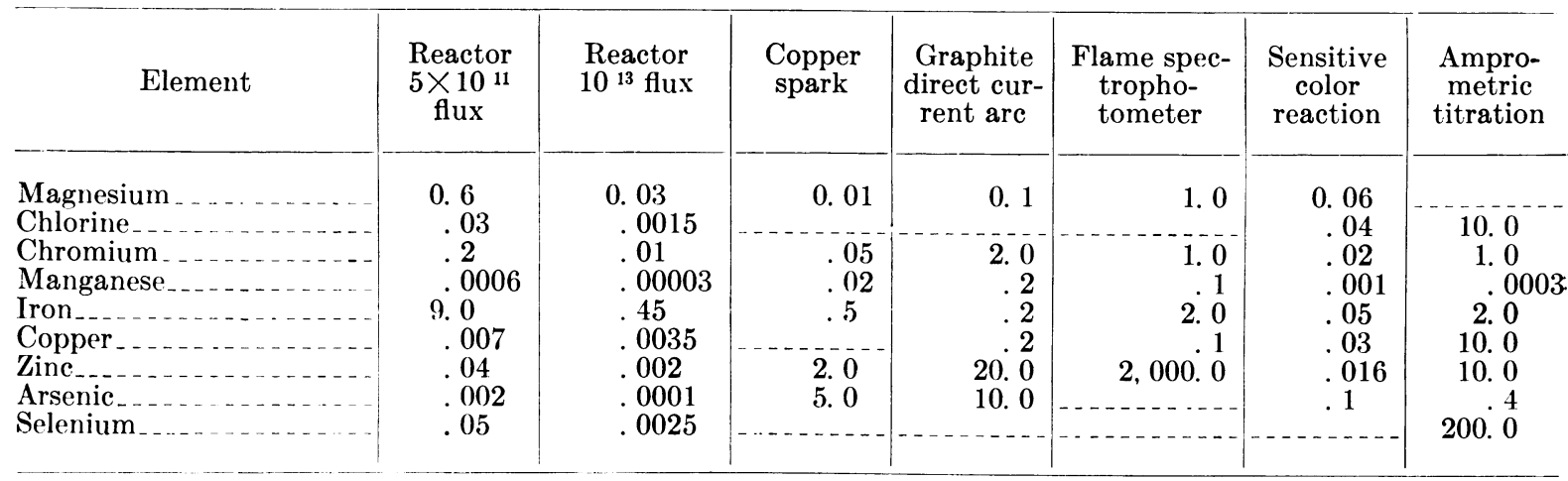

the isotope under investigation as a result of the decay of other radioactive species. However, this limitation may be overcome by determining decay and energy of emission.

\section{Comparison With Other Methods}

Meinke (6) has compared activation analysis with other means of trace analysis for elements from hydrogen to uranium. His findings on a few elements of importance in drinking water standards are given in table 2. For all of these except iron and magnesium, neutron activation analysis at a thermal neutron flux of $10^{13}$ is more sensitive than any of the other trace methods considered.

\section{Its Future in Public Health}

We believe that activation analysis has a future in public health laboratory operations. Sensitivity of the method is increasing wit', the development of higher flux reactors. In fact, Meinke reports that many scientists feel that activation analysis will be the ultimate method of analysis. Its use, of course, depends on the availability of nuclear reactors.

We suggest that the reader become more familiar with the method through the references given $(2,5-8)$ and determine whether it is a possible solution to his problem. An activation analysis service is presently available at the Oak Ridge National Laboratory for a nominal charge to industrial, academic, and govern- mental institutions. Samples are sent to G. W. Leddicotte, Analytical Chemistry Division, Oak Ridge National Laboratory, P. O. Box P, Oak Ridge, Tenn. Following analysis, the results are returned to the sender in terms of micrograms per gram, parts per million, or other usable units, as requested. Additional information may be obtained by writing to the above address.

\section{REFERENCES}

(1) Hevesy, G., and Levi, H.: Action of neutrons on the rare earth elements. Kgl. Danske Videnskab. Selskab-Math.-fys. Medd. 14: 1-34 (1936).

(2) Leddicotte, G. W., and Reynolds, S. A.: Neutron activation analysis. Oak Ridge National Laboratory CF-55-11-20. Oak Ridge, Tenn., 1955.

(3) Hollander, J. M., Perlman, I., and Seaborg, G.: Table of isotopes. Rev. Mod. Phys. 25: 469-651 (p. 613), April 1953.

(4) Kahn, B.: Abstracts of unclassified methods for the separation of radionuclides. Oak Ridge, Tenn., Oak Ridge National Iuaboratory, Waste Disposal Research Section, 1954. Processed.

(5) Smales, A. A. : The scope of radioactivation analysis. Atomics 4: 55-63, 74, March 1953.

(6) Meinke, W. W. : Trace-element sensitivity : Comparison of activation analysis with other methods. Science 121: 177-184, Feb. 11, 1955.

( 7 ) Leddicotte, G. W., and Reynolds, S. A. : Activation analysis with the Oak Ridge reactor. Nucleonics 8: 62, March 1951.

(8) Brooksbank, W. A., Leddicotte, G. W., and Mahlman, H. A.: Analysis for trace impurities by neutron activation. J. Phys. Chem. 57: 737863, November 1953. 